\title{
CASE OF POST COVID PULMONARY MUUCORIMYCOSIS
}

KEY WORDS:

\section{Dr.Chetan Prajapati}

\section{Dr.Jagruti Ahir*}

Dr. Ghanshyam

\section{Borisagar}

\section{Dr.Madhavi} Dhameliya

\section{$3^{\text {rd }}$ Year Resident, pulmonary Medicine ,B.J.Medical College Ahmedabad.}

$3^{\text {rd }}$ Year Resident, pulmonary Medicine ,B.J.Medical College Ahmedabad. *Corresponding Author

Associate Professor, Department of Pulmonary Medicine, B.J Medical College Ahmedabad.

Assistant Professor, Department of Pulmonary Medicine, B.J Medical College Ahmedabad.

Pulmonary mucormycosis is a relatively rare pulmonary fungal disease, which is difficult to diagnose early and lacks
effective treatment. It is seen in patients with hematological malignancies, diabetes mellitus, and immunocompromised
states. The diagnosis depends primarily on the detection of fungi in lung tissue. Here, we present a case of a 54-year-old
male who has type 2 diabetes mellitus and a past history of treated covid positive LRTI. Clinical diagnosis is difficult in
pulmonary mucormycosis, and early diagnosis is needed for this life-threatening infection. Histopathological
examination of a resected cavity confirmed the diagnosis of pulmonary mucormycosis. This report highlights the
difficulty of diagnosis and the importance of histological examination in detecting mucormycosis which will help for
early management.

\section{INTRODUCTION}

Mucormycosis is a rare opportunistic fungal infection. It derives its name from the Mucorales order of filamentous fungi and family Mucoraceae. It is a less common opportunistic fungal infection compared to Candida and Aspergillus species [1]. There are six most commonly reported forms which include rhinocerebral, pulmonary, cutaneous, gastrointestinal, disseminated, and uncommon presentations [2, 3]. The relative incidence of pulmonary mucormycosis (PM) to other clinical form incidence in literature is about 25\% [4]. Diabetes mellitus, systemic corticosteroid therapy, neutropenia, hematologic malignancies, stem cell transplant, and immuno compromised state are the predisposing situations for mucormycosis $[3,4]$. Pulmonary mucormycosis results from the inhalation of sporangiospores or by hematogenous or lymphatic spread [3, 5]. Patients present with nonspecific symptoms like cough, dyspnea, chest pain, and fever $[3,5]$. Clinical diagnosis is difficult in pulmonary mucormycosis, and early diagnosis is needed for this lifethreatening infection [6]. There are no reliable serological, PCR-based, or skin tests for mucormycosis. Sterile culture does not rule out the diagnosis. Histopathology and direct microscopy along with culture from various clinical specimens except blood are the major diagnostic modalities for mucormycosis [5

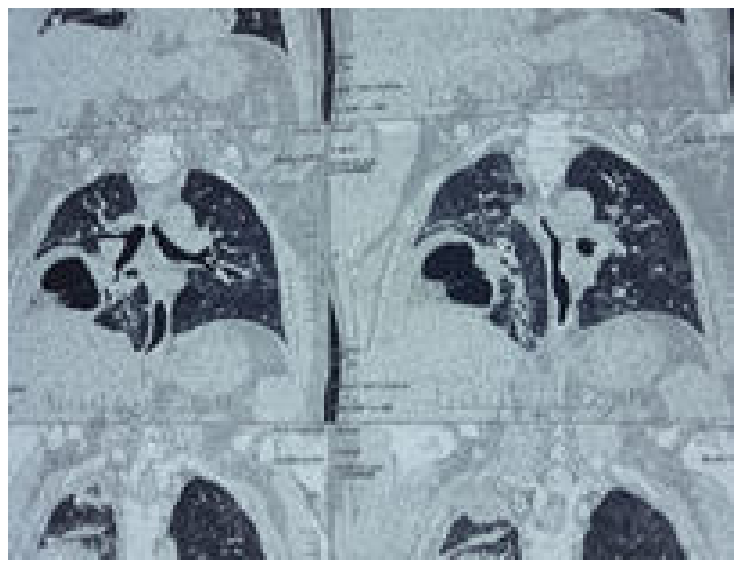

Figure 1: CT showing cavitation of the right lower lobe. |www.worldwidejournals.com|

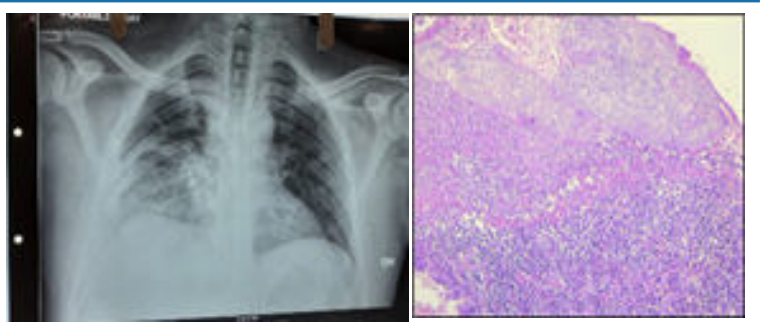

Figure 2: Chest radiograph, PA view showing cavitary lesion in the right lower lobeFigure 3: Lung tissue with cavity containing fungal ball (arrow), H\&E 20x.

\section{CASE REPORT}

A 54-year-old male Patient was RT PCR positive on 17 april 2021 CTSS 20/25 patient was admitted at that time for 10 days and had taken inj remedesivir and inj steroids patient was newly diagnosed DM 2 at that time.After lmonth patient presented to the emergency department with a complaint of cough with whitish and brownish expectoration ,fever on off since 15 days with past complain of massive hemoptysis before 17 days for which CTPA was done at zydus hospital at that time s/o right pulmonary artery lower lobar branch pseudo aneurysm detected and embolization was done. No history of weight loss or night sweats. General physical examination was insignificant. . Laboratory investigations showed a hemoglobin of $12 \mathrm{gm} / \mathrm{dl}$, total WBC count of 7,300 cells/cumm, and platelets 4.02 lakhs/cumm. RFT and LFT were normal Local examination revealed right lower lobe crepitation.

chest radiograph (Figures 1 and 2) revealed right lower lobe thick wall cavitory lesion. HRCT thorax s/o :well-defined, hypodense, nonenhancing lesion is noted in the cavitation that was reported as a mucous plug or a fungus ball. There was no endobronchial lesion or mucosal abnormalities. There was a cavity in the right lower lobe. Bronchoalveolar lavage analysis showed inflammatory cells, macrophages, and degenerated cells. There was no evidence of malignancy. Bronchoalveolar lavage Culture was done s/o aseptate with variable branching and wide angle bifurcating fungal hypae in favour of mucour fungus. 
We have started injectable lyophilized amphotericin B 5mg $/ \mathrm{kg}$ IV q $24 \mathrm{~h}$ in dextrose $5 \%$ solution and oral tablet of posaconazole $300 \mathrm{mg}$ BD on first day and then $300 \mathrm{mg}$ OD from next day onward . Patient was afebrile after 7 days. We have given inj amphotericin for 21 days with renal function monitoring every 2 days.Then patient was discharged with oral posaconazole and advise to follow up after 15 days. On follow up after 15 days patient was clinically and radiologically improved we have continued tab posaconazole for more 3 months.

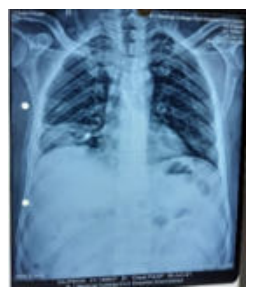

\section{AFTER AMPHOTERICIN GIVEN}

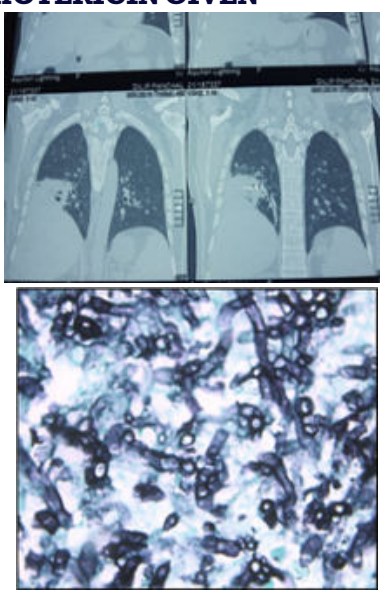

Figure 5: Broad nonseptate hyphae with right-angled branching, Gomori's methenamine silver (GMS), 100x.

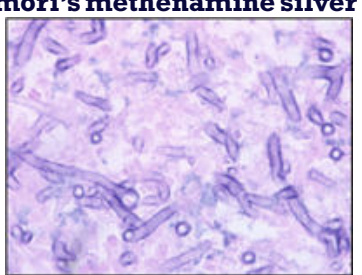

Figure 6: Broad nonseptate hyphae with right-angled branching, H\&E 100x.

\section{DISCUSSION}

Mucormycosis encompasses a group of infections caused by the fungi belonging to the order Mucorales and family Mucoraceae [1,7].Rhizopus oryzae is the most common cause of infection from the Mucoraceae family followed by Mucor sp. and Lichtheimia sp. Inhalation of ubiquitous spores is the main mode of infection. There is no evidence of human to human transmission [8]. Recent reclassification has abolished the class zygomycetes, and hence, the term zygomycosis is inappropriate for use, although it may still be used to address mucormycosis [1]. Pulmonary mucormycosis is a devastating and life-threatening infection if not correctly diagnosed and treated [9]. The mononuclear and polymorphonuclear phagocytes of normal hosts kill Mucorales by generation of oxidative metabolites and the cationic defensins. Defensive mechanisms in normal hosts are mediated by macrophages that inhibit germination of spores and neutrophils that kill hyphal elements by oxidative burst. Macrophage dysfunction is the cause for infection in diabetes patients [10]. Weak host defenses are a major risk factor for pulmonary mucormycosis. Neutropenic patients as well as patients with elevated serum iron levels are also at increased risk of developing mucormycosis [5].
Pulmonary mucormycosis may develop as a result of inhalation of spores or by hematogenous or lymphatic spread. Portal of entry for Mucorales is the respiratory tract where the fungi can easily invade arteries, veins, and lymphatics and produce thrombosis and infarction which can be fatal [11-13]. Invasive pulmonary mucormycosis causes severe morbidity and mortality in patients with hematological malignancies, diabetes mellitus, hematopoietic stem cell transplant and solid organ transplant patients, patients on corticostero idbased therapy, iron overload and chelation therapy, intravenous drug use, trauma, burns, neonatal prematurity, and malnutrition [14-19].The sequelae include angioinvasion and direct tissue injury of the respiratory tract, direct extension from the lungs into the great vessels, invasion from the paranasal sinuses into the orbit and brain, and hematogenous dissemination to the central nervous system $[14,20]$. Clinical presentations may include nonspecific symptoms like fever, dyspnea, cough, and chest pain, and rarely, they can present as progressive subcutaneous emphysema, Pancoast syndrome, Horner's syndrome, and bronchial perforation [21]. Angioinvasion results in necrosis of parenchymal tissue, cavitation and/or hemoptysis which may be fatal if a major blood vessel is involved [22].

In a large review of 929 cases of zygomycosis, an overall mortality of $44 \%$ was reported in diabetics with zygomycosis and $76 \%$ mortality for pulmonary zygomycosis in patients with other predisposing factors. Rhizopus species were the most common organism in patients of zygomycosis in whom hematological malignancies were the predisposing factor [16].

Histopathology and direct microscopy along with culture for various clinical specimens are the major diagnostic modalities for mucormycosis. Sputum and BAL cytology are unpredictable and may be negative $[10,23]$. Histopatho logically, mucormycosis species appear as broad, nonseptate hyphae with right angle branching and can be differentiated from Aspergillus which shows regular, septate, and acute angle branching hyphae [24].

Radiological findings described in literature are the presence of nodule or consolidation, isolated mass, cavitation, or lung abscess with fungal ball. Wedge-shaped infarcts of the lung may also be seen, particularly following thrombosis of the pulmonary vessels due to fungal angioinvasion. Highresolution CT scan is the best method of determining the extent of pulmonary mucormycosis and may demonstrate evidence of infection. Expansion of a mass or consolidation across tissue planes towards the great vessels in the mediastinum may suggest the diagnosis [25, 26]. "Reverse halo sign" is described as a prominent CT finding present in the early course of disease [5].

Differential diagnosis of PM involves bacterial, viral, and other fungal pneumonias and invasive fungal infections can be a diagnostic challenge [27]. Invasive PM needs to be distinguished from invasive aspergillosis as the two respond differently to antifungal agents [24]. Confirmation by microscopic examination is necessary as treatment will differ. In our case, before confirmation by histopathology, the patient was put on posaconazole, and after confirmation, it was changed to liposomal amphotericin B. Rarely, PM may recur even in an immunocompetent host [28]. Lipid formulation of amphotericin B is the first-line therapy for mucormycosis [29].

\section{CONCLUSION}

Mucormycosis is an opportunistic life-threatening infection mostly occurring in the immunocompromised host. The risk factors are many including hematological malignancies, uncontrolled diabetes mellitus, and immunocompromised states. Rhino-orbito-cerebral is the most predominant form of clinical presentation. Diagnosis is difficulty due to nonspecific presentation. Early diagnosis and treatment 
which includes surgery and antifungal drugs can improve outcome and survival. Definitive diagnosis required pathologic demonstration of the organism in affected tissue as sputum and BAL culture rarely shows growth. The best promising diagnostic modalities should be applied at earliest to avoid unnecessey treatment.

\section{Conflicts of Interest}

The authors declare that there is no conflict of interest regarding the publication of this paper.

\section{REFERENCES}

[1] B. Spellberg and J. Maertens, "Mucormycosis," in Principles and Practice of Transplant Infectious Diseases, A. Safdar, Ed., Springer, New York, NY,2019.

[2] E. Pennell, I. Pecson, C. Nakamura, and A. E. Galvis, "Pulmonary Cases, vol. 14, p.e00474,2018.

[3] A. Serris, F. Danion, and F. Lanternier, "Disease entities in mucormycosis," Journal of Fungi, vol.5, no. 1, p. 23,2019.

[4] Z. Luo and L. Zhang, "Diagnosis and treatment of pulmonary mucormycosis: a case report," Experimental and Therapeutic Medicine, vol. 14, no. 4, pp. 3788$3791,2017$.

[5] B. Spellberg, J. Edwards, and A. Ibrahim, "Novel perspectives on mucormycosis: pathophysiology, presentation, and management," Clinical Microbiology Reviews, vol. 18,no.3,pp.556-569,2005.

[6] B.Nam, T.J.Kim, K.S. Lee, T. S. Kim, J.Han, and M.J. Chung, European Radiology, vol.28, no. 2, pp. 788-795,2018.

[1] A.Mohammadi,A.Mehdizadeh,M.Ghasemi-Rad, H. Habibpour, and A. Esmaeli, "Pulmonary mucormycosis in patients with diabetic ketoacidosis: a case report and review of literature," Tuberk Toraks, vol.60, no. 1,pp.66-69,2012.

[8] S. Point, F. Gabriel, H. Bégueret et al., "Tumor shape pulmonary mucormycosis associated with sinonasal aspergillosis in a diabetic patient," Medical microbiology case reports, vol. 19,pp. 13-17,2018.

[9] N. Iqbal, M. Irfan, K. Jabeen, M. M. Kazmi, and M. U. Tariq, "Chronic pulmonary mucormycosis: an emerging fungal infection in diabetes mellitus," Journal of Thoracic Disease, vol. 9, no.2,pp.E121-E125,2017.

[10] J. Guarner and E. Brandt, "Histopathologic Diagnosis of fungal infections in the

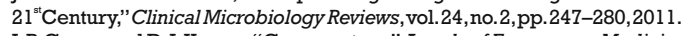

[11] J.P. Green and D. J. Karras, "Commentary," Annals of Emergency Medicine, vol. 59, no. 1,pp. 54-55, 2012.

[12] H. S. Yamin, A. Y. Alastal, and I. Bakri, "Pulmonary mucormycosis over 130 years: a case report and literature review," Turkish Thoracic Journal, vol. 18, no. 1, pp. 1-5,2017.

[13] G. Helenglass, J. A. Elliott, and N. P. Lucie, "An unusual presentation of opportunistic mucormycosis," British Medical Journal (Clinical Research Ed.), vol.282, no. 6258,pp. 108-109, 1981. Case Reports in Pathology

[14] T. J. Walsh, M. N. Gamaletsou, M. R. McGinnis, R. T. Hayden, and D. P. Kontoyiannis, "Early clinical and laboratory diagnosis of invasive pulmonary, extrapulmonary, and disseminated mucormycosis (zygomycosis)," Clinical Infectious Diseases, vol.54,suppl_1,pp.S55-S60,2012.

[15] G. M. Cox, "Mucormycosis (zygomycosis)," 2019, Literature review current through:UpToDate.Last updated.

[16] M.M.Roden,T.E.Zaoutis,W.L. Buchanan et al. "Epidemiology and outcome of Zygomycosis: a review of 929 reported cases," Clinical Infectious Diseases, vol. 41, no. 5,pp. 634-653,2005.

[17] H. C. Pillsbury and N. D. Fischer, "Rhinocerebral mucormycosis," Archives of Otolaryngology, vol. 103, no. 10, pp.600-604,1977.

[18] J. W. Tomford, D. Whittlesey, J. J. Ellner, and J. F. Tomashefski Jr., "Invasive primary cutaneous phycomycosis in diabetic leg ulcers," Archives of Surgery, vol.115, no. 6,pp.770-771,1980.

[19] M. Tedder, J. A. Spratt, M. P. Anstadt, S. S. Hegde, S. D. Tedder, and J. E. Lowe, "Pulmonary mucormycosis: results of medical and surgical therapy," The Annals of Thoracic Surgery, vol. 57, no. 4,pp. 1044-1050, 1994.

[20] P. Khatiwada, A. Giri, and P. Khatiwoda, "Mucormycosis in diabetes mellitus," Journal of Advances in Internal Medicine, vol. 1, no. 2,pp. 73-75, 2012.

[21] X.-M.Wang, L.-C. Guo, S.-L. Xue, andY.-B. Chen, "Pulmonary mucormycosis: a case report and review of the literature," Oncology Letters, vol. 11, no. 5, pp. 3049-3053, 2016.

[22] R. Garg, R. S. K. Marak, S. K. Verma, J. Singh, Sanjay, and R. Prasad, "Pulmonary mucormycosis mimicking as pulmonary tuberculosis: a case report," Lung India, vol.25, no.3, pp. 129-131,2008.

[23] S. Challa, S. G. Uppin, M. S. Uppin, R. T. Paul, A. K. Prayaga, and M. T. Rao, "Pulmonary zygomycosis: a clinicopathological study," Lung India, vol. 28, no. 1,pp. 25-29,2011.

[24] J. G. Song, S. H. Kang, B. W. Jung, H. S. Oh, M. J. Kim, and S. H. Lee, "Invasive pulmonary aspergillosis histologically mimicking mucormycosis," The Ewha Medical Journal,vol.39, no.2,pp.65-68,2016.

[25] M. C. Fitzpatrick and B.W. Carter, "Pulmonary mucormycosis complicating cutaneous blastic plasmacytoid dendritic cell neoplasm," Baylor University Medical Center Proceedings, vol. 25, no. 3,pp. 287-288, 2012.

[26] H. P. McAdams, M. Rosado de Christenson, D. C. Strollo, and E. F. Patz Jr., "Pulmonary mucormycosis: radiologic findings in 32 cases," American Journal of Roentgenology, vol. 168, no. 6, pp. 1541-1548, 1997.

[27] H. Tanriverdi, F. Atalay, L. Kart, M. Altuntas, and A. Tomruk, "A case of pulmonary mucormycosis presenting with endobronchial involvement," Eurasian Journal of Pulmonology, vol. 16, no. 3,pp. 189-191,2015.

[28] L. Zhang, X. Tian, P. Wang, H. Zhang, and R. Feng, "Recurrent pulmonary mucormycosis after lobectomy in a non-smoking patient without predisposing risk factors," The Brazilian Journal of Infectious Diseases, vol. 16, no. 6,pp. 590-593,2012.

[29] A. Skiada, C. Lass-Floerl, N. Klimko, A. Ibrahim, E. Roilides, and G. Petrikkos, "Challenges in the diagnosis and treatment of mucormycosis," Medical Mycology,vol.56,suppl_1,pp.S93-S101,2018. 